Ann. Sci. forest., 1980, 37 (4), 381-386.

\title{
Rapport synthétique des discussions et débats du colloque
}

\author{
R. PERRIN \\ Laboratoire de Pathologie forestière, \\ Centre national de Recherches forestières, I.N.R.A., \\ Champenoux, 54280 Seichamps
}

Le débat, souvent très animé, qui s'est instauré à l'issue de chaque communication, s'esî révélé très fructueux.

\section{La maladie de l'écorce du hêtre aux U. S. A.}

La présence de D. R. Houston a permis d'évoquer les circonstances particulières de l'évolution de cette maladie aux Etats-Unis. Elle poursuit sa lente extension vers le Nord-Est. On ne recense aucune mortalité au niveau du front de progression, dans les zones, malheureusement très peu étudiées, où la cochenille est seule présente. Les progressions de l'insecte et du champignon paraissent simultanées. S'il est bien établi que la cochenille a été introduite sur ce continent, l'existence et la persistance de $N$. coccinea var. faginata en l'absence de cochenille, sonł encore controversées. Dans la zone résiduelle, il subsiste de rares sujets considérés comme résistants. La variabilité considérable du hêtre américain (Fagus grandifolia) se traduit par l'existence de nombreuses variétés. La connaissance de leur comportement vis-à-vis de la maladie de l'écorce du hêtre est encore imparfaite, d'autant que certaines variétés n'existent qu'en dehors de la zone atteinte. Cependant, on rencontre toutes les transitions entre la sensibilité extrême et la résistance totale. La faculté de reproduction de $F$. grandifolia par drageons en fait un matériel de choix pour l'étude des variations de sensibilité à la maladie.

Enfin, la présence de Fagus sylvatica, à titre d'arbre d'ornement aux Etats-Unis, autorise la comparaison de l'effet de $N$. coccinea et sa var. faginata sur les deux espèces de Fagus.

La lente agonie du seul exemplaire de Fagus grandifolia de l'arboretum d'Amance (M.-et-M., France), résulte d'une infection de l'écorce par N. coccinea après $C$. fagisuga.

La discussion a permis de préciser certaines étapes et de souligner les phases fondamentales du phénomène parasitaire.

La prédisposition de l'arbre aux infestations de $C$. fagisuga ou à l'action destructrice de $N$. coccinea, n'est pas démontrée expérimentalement, mais fortement suggérée par de multiples observations. Il s'agit vraisemblablement d'un événement capital dans le déroulement de la maladie, mais nos connaissances limitées en ce domaine ne permetlent pas de préciser les facteurs qui concourent à une prédisposition. Les phé- 
nomènes interférant avec la nutrition de l'arbre, ou altérant, même partiellement ou momentanément, le fonctionnement du système racinaire, souvent ignorés, constitueraient la raison essentielle de l'établissement d'un état de prédisposition.

\section{Syndrome}

Cryptococcus fagisuga est l'élément primaire de ce complexe maladif, assurant parfois la dissémination de $N$. coccinea. La dispersion de l'insecte à courte distance seulement entraîne la constitution d'agrégations d'arbres atteints, au milieu desquels certains arbres indemnes sont considérés comme résistants. Cette aptitude pourrait être étudiée à partir de descendances actuellement disponibles en Angleterre.

Pour se nourrir l'insecte plonge ses stylets dans la partie externe de l'écorce, atteignant rarement le cambium, jusqu'où diffusent cependant les sécrétions de la cochenille, et notamment des substances de croissance. Dans la mesure où la réaction de l'écorce à différentes sortes de blessure n'est pas spécifique, l'apparition de suintements, à la suite du traitement au 2-4-5 T, à des concentrations élevées, ne permet pas de conclure. La diffusion de la substance de croissance dans l'écorce engendre un affaiblissement, certes plus accusé, mais comparable à celui dont profite N. coccinea après les piqûres de $C$. fagisuga. Cette perturbation physiologique, particulièrement évidente localement, mais également sensible globalement, offre à $N$. coccinea un avantage déterminant, vraisemblablement temporaire, s'ajoutant aux changements anatomiques, considérés comme définitifs (Braun, 1976). Dans ce phénomène réside l'explication de la réussite d'inoculations artificielles de $N$. coccinea ou de la progression de la nécrose pendant la saison de végétation, en présence de fortes infestations de cochenille, associées à une réaction amoindrie de l'arbre. L'infection par N. coccinea peut intervenir même si l'insecte est rare, mais l'évolution ultérieure de la lésion dépend de l'abondance des colonies. Quelques arbres survivent en dépit d'une forte infestation de cochenille, ou se rétablissent, malgré l'initiation de lésions corticales, à la suite d'une régression plus ou moins brutale de la cochenille. L'arbre conserve les traces de ces attaques, sous la forme d'une écorce fissurée, ou, en présence de $N$. coccinea, d'une plaie chancreuse présentant une écorce excessivement perturbée ef très crevassée. Le champignon se retrouve toujours dans les lésions de l'écorce trahies par un suintement, s'il est associé ou précédé par la cochenille. Par contre, il peut être absent des lésions révélées par des écoulements consécutifs à des événements (sécheresse par exemple), où la cochenille n'intervient pas.

Le bénéfice tiré par les champignons lignivores de la présence préalable de $N$. coccinea, prouve que, par leur intervention précoce, ils contribuent, de manière importante, à la dégradation de l'écorce.

La prédominance de certaines espèces ( $F$. fomentarius en Europe continentale, Bjerkandera adusta en Angleterre) trouve ses raisons dans certaines influences climatiques, mais dépend aussi de l'âge et de la taille de l'arbre. Les galeries de scolytes favorisent une rapide invasion du bois par les lignivores.

\section{Facteurs du milieu}

La gravité de la maladie, dont la recrudescence est générale en Europe consécutivement aux extrêmes climatiques de 1976, dépend des conditions stationnelles. 
Importante sur les sols «lourds», argilolimoneux, elle est négligeable sur les sols siliceux. Les conséquences des fluctuations climatiques, ou de l'intervention de parasites comme Phyfophthora sp., seraient plus accusées dans certaines situations.

La nécessité d'éclaircies sanitaires précoces est indéniable. Aux Etats-Unis cependant, la régénération par drageons favorisée par l'abattage des arbres malades, la moindre valeur économique du hêtre comparée à celles des espèces associées (Acer saccharum, Befula alleghaniensis) rend l'intérêt des éclaircies sanitaires plus discutable.

L'éclaircie n'a aucune influence sur l'évolution des populations de cochenille, alors qu'elle réduit sensiblement les infections de $N$. coccinea. Cette intervention sylvicole modifie les relations arbre/insecte/champignon. La compétition accrue dans les peuplements non éclaircis accuserait l'affaiblissement provoqué par la cocheniile ef dont le champignon tire profit.

\section{La lutte biologique}

Les différentes observations s'accordent pour constater l'effet négligeable des prédateurs de l'insecte. Ceux-ci, dont la coccinelle est le représentant le plus fréquent, ont eux aussi leurs propres prédateurs. Les relations intervenant dans cette chaîne trophique sont d'une extrême complexité.

Dans certaines circonstances, la réaction du hêtre à la présence d'Ascodichaena rugosa, champignon épiphyte, consiste en la formation d'un périderme secondaire, s'opposant à l'installation de la cochenille. Cette particularité pourrait être exploitée, en favorisant l'installation et le développement du champignon, par le maintien d'une ambiance humide dans la hêtraie. Remarquons cependant que l'éclaircie en réduisant l'humidité au niveau des fûts produit l'effet inverse.

La session de clôture (soirée du 10 mai ef matinée du 11 mai) a consacré les progrès, incontestables, accomplis depuis 1970 dans nos connaissances, principalement dans le domaine étiologique, où se distinguent clairement les différents événements engendrant la destruction de l'écorce du hêtre. La compréhension du phénomène maladif souffre encore de lacunes, particulièrement dans les conditions écologiques favorables au déclenchement ou déterminant l'ampleur de la maladie.

\section{Etiologie}

L'association spécifique entre Cryptococcus fagisuga et Nectria coccinea constitue l'ossature du phénomène parasitaire, diversifiant son expression en fonction des conditions écologiques locales (âge, structure sylvicole, ...). Les interactions hêtre/ insecte/champignon qui règlent l'évolution de la maladie, subissent des fluctuations temporelles et les influences du milieu encore mal comprises. Cette situation découle, vraisemblablement, des difficultés d'expérimentation. Elle a pour corollaire, l'embarras rencontré dans certains cas pour apprécier le stade d'évolution de la maladie. 
La cause principale de l'altération de l'écorce du hêtre, résulte de l'association C. fagisuga/N. coccinea, mais d'autres circonstances ou événements aboutissent à des syndromes voisins, dont le symptôme le plus commun est le suintement. La sécheresse, les températures extrêmes, les maladies racinaires engendrent des lésions corticales parfois limitées, plus ou moins superficielles, qui se cicatrisent quelquefois. Les conditions favorables à une perfurbation de la «physiologie» du hêtre sont nombreuses. Elles créent une prédisposition aux parasites de faiblesse dont $N$. coccinea est le plus fréquent. Bien qu'intervenant tardivement dans la chaîne parasitaire, il est constaminent présent dans la hêtraie sous phase saprophytique.

\section{La cochenille}

L'influence de l'état physiologique de l'arbre sur les pullulations de l'insecte est encore controversée. La prédisposition de quelques arbres aux attaques de cochenille est suggérée par certaines observations, mais une véritable démonstration expérimentale fait défaut. Un des principaux obstacles est l'absence de critère permettant de caractériser l'étal physiologique de l'arbre.

La plus ou moins bonne résistance à la cochenille, dont l'expression interfère avec la prédisposition de l'hôie, est par contre bien établie. Malgré la difficulté de transposer aux feuillus les observations relatives aux résineux, certains auteurs mentionnent le cas d'adaptation de cochenilles ou de pucerons à la physiologie de certains Pinus, marquant ainsi la complexité des relations arbre/insecte.

De nombreuses questions demeurent irrésolues, ef ces lacunes ont éfé d'autant plus ressenties, qu'elles concernent la phase essentielle du phénomène. En quoi les facteurs du milieu font-ils pencher les relations hêtre/cochenille en faveur de l'un ou de l'autre, dans un contexte sylvicole donné ? Quelle est la dynamique des populations de cochenille? Quelles sont les répercussions d'un stress subi par l'hôte sur les populations de l'insecte? De quelle nature sont les changements induits dans l'écorce par l'insecte? En quoi sont-ils favorables à Nectria coccinea ? Ce domaine, quoique fondamental, reste pratiquement inexploré.

\section{Le champignon}

En plus de l'existence d'une voie de pénétration, ce parasite de faiblesse, pour se manifester, nécessite un affaiblissement marqué de l'hôte, provoqué dans la majorité des cas par $C$. fagisuga. A partir de quelle densité de colonies de cochenille, l'infection, puis l'extension de la lésion se réalisent-elles? La part prise par $N$. coccinea dans les autres affections de l'écorce, où n'intervient pas la cochenille, est encore méconnue. Les connaissances relatives à la variabilité du pouvoir pathogène du champignon en relation avec la vigueur de l'arbre font défaut.

\section{Influence de l'environnement}

La recrudescence, plutôt que le déclenchement, de la maladie avec la sécheresse de l'année 1976, a été générale en Europe. Cette influence climatique, brutale et 
prolongée, est à l'origine d'un affaiblissement des arbres, particulièrement marqué sur certaines stations.

La situation particulière de l'Amérique du Nord est celle qui se prêfe le mieux à l'étude de diverses influences. En avant du front de progression, la variété des conditions stationnelles, microclimatiques, sylvicoles, ... promet des observations fructueuses sur les circonstances favorables ou défavorables à la manifestation épidémique de la maladie.

Quelles sont les conséquences des attaques actuelies sur les peuplements à venir? Aux U.S. A., la multiplication par drageons privilégie la descendance des arbres sensibles. En Europe au contraire, la morł des sujets sensibles avant leur reproduction laisse espérer que les peuplements issus de régénération naiurelle seront globalement moins vulnérables.

\section{Les implications pratiques}

L'insuffisance des connaissances sur les effets de l'éclaircie dans le déclenchement et l'évolution de la maladie de l'écorce du hêtre, ne permet pas de conseiller cette pratique sylvicole pour atténuer les pertes. L'éclaircie demeure avant tout une intervention sylvicole. Cependant, dans un massif où sévit la maladie, le marteau portera de préférence sur les plus gros sujets qui, plus éprouvés, lors de conditions adverses, se révèlent plus fréquemment afteints.

La principale justification de l'éclaircie sanitaire est la volonté de ne rien perdre sur la valeur marchande de l'arbre. On ne peut la créditer que d'une réduction négligeable des risques d'infection. L'ampleur et la précocité de l'intervention doivent être l'aboutissement d'un calcul économique tenant compte des pertes subies et des répercussions sur l'avenir du peuplement.

La télédétection constitue un moyen de prévision très efficace, malheureusement prohibé par son prix de revient élevé ; elle ne pourrait être envisagée que dans le cadre d'une mission conciliant plusieurs objectifs différents dans une même région.

\section{Les besoins en recherche}

Les études de la dynamique de la maladie en relation avec les différentes composantes du milieu (âge, station, type de sylviculture, déséquilibre nutritif, alimentation hydrique, ...) doivent être poursuivies ou engagées en priorité.

Deux approches, complémentaires, sont envisageables :

- l'une expérimentale et scientifique, consiste en un examen critique et déłaillé des relations entre le hêtre et la cochenille en contrôlant le maximum de facteurs du milieu :

- l'autre, pratique, suppose la création de placettes d'observation construites sur un même modèle (transect), installées en fonction d'objectifs déterminés, dans de multiples situations. Un système de notation commun autorisera la comparaison des différentes observations effectuées. Ce réseau répondra parfaitement à la nécessité, ressentie par tous, d'élargir les investigations à l'échelle du continent. 


\section{Conclusion}

L'intérêf des communications, l'animation des débats, la contribution active ef spontanée des participants à une réflexion approfondie, aftestent du succès et de l'opportunité du colloque sur la maladie de l'écorce du hêtre.

La clarification d'une étiologie complexe, la révélation de phénomènes essentiels comme la résistance à la cochenille, marquent une incontestable progression de nos connaissances. Mais le résultat, certainement le plus fructueux, est d'avoir pu délimiter les domaines imparfaitement explorés, et définir les moyens de les mieux pénétrer.

En provoquant la création d'un groupe I. U. F. R. O. spécialisé (D. R. Houston Chairman, et $D$. Wainhouse Cochairman), le colloque a jeté les bases d'une véritable coopération internationale, préalable nécessaire à une évolution rapide de nos connaissances dans l'avenir. La détermination des besoins en recherche, l'harmonisation des programmes, préparent la coordination de tous les efforts vers une stratégie de lutte commune dont la mise au point à moyen terme n'est plus simple illusion.

\section{Remerciements}

Malgré les difficultés et l'inévitable subjectivité inhérentes à l'établissement de telles rédactions, l'incomparable richesse, les nombreux enseignements qui se dégagent des discussions m'ont conduit à en rédiger une synthèse. Je tiens à remercier particulièrement $E$. J. Parker pour ses notes détaillées qui m'ont été d'une grande utilité.

Reçu pour publication en décembre 1979. 